\title{
Perceived Presence's Role on Learning Outcomes in a Mixed Reality Classroom of Simulated Students
}

\author{
Aleshia T. Hayes ${ }^{1}$, Stacey E. Hardin ${ }^{2}$, and Charles E. Hughes ${ }^{3}$ \\ ${ }^{1}$ University of Central Florida: Synthetic Reality Lab., Institute for Simulation and Training, \\ Orlando, FL, USA \\ ${ }^{2}$ University of Central Florida - College of Education, Orlando, FL, USA \\ ${ }^{3}$ University of Central Florida: Synthetic Reality Lab, Computer Science Division, \\ Orlando, FL, USA \\ \{Aleshia.prof, Staceyehardin\}@gmail.com, ceh@cs.ucf.edu
}

\begin{abstract}
This research is part of an ongoing effort on the efficacy and user experience of TLE TeachLivE ${ }^{\mathrm{TM}}$, a 3D mixed reality classroom with simulated students used to facilitate virtual rehearsal of pedagogical skills by teachers. This research investigated a potential relationship between efficacy, in terms of knowledge acquisition and transfer, and user experience in regard to presence, suspension of disbelief, and immersion. The initial case studies examining user experience of presence, suspension of disbelief, and immersion were used to develop a presence questionnaire revised from the work of Witmer and Singer (1998) to address the TLE TeachLivE ${ }^{T M}$ mixed reality environment. The findings suggest that targeted practice, authentic scenarios, and suspension of disbelief in virtual learning environments may impact learning.
\end{abstract}

Keywords: Mixed Reality Classroom, Simulation, Presence, Suspension of Disbelief, Immersion, Engagement, Knowledge Acquisition, Virtual Learning.

\section{Introduction}

\section{$1.1 \quad$ The Environment}

The research reported here is part of a large scale study at the University of Central Florida investigating the efficacy and user experience of TLE TeachLivETM, a 3D mixed reality classroom with five simulated students, used to facilitate virtual rehearsal of pedagogical skills in pre-service and practicing teachers. The classroom consists of five students cognitively and behaviorally modeled after research-based student archetypes whose avatars are visually modeled to be representative of diverse middle school populations. The classroom and students are displayed on a large screen high definition display and the current interface tracks user movement allowing the teacher's physical movement to be reflected by changes in the perspective position of the virtual camera. This creates a relative point of view, which is 
reflective of his or her physical proximity and orientation in relation to the students and the classroom environment. The virtual students, through human in the loop simulation, interact with each other and with the teachers. Teachers move freely in the environment to interact with students in the simulated classroom as they deliver practice lessons. The student avatars respond to teacher's questions, behaviors, classroom strategies and lessons in ways that are authentic to each student's characteristics and learning styles as well as the pedagogical delivery of the teacher.

\subsection{System Function}

Teachers enter the simulator and virtually rehearse pedagogical or content driven objectives. Immersed in TLE TeachLivE ${ }^{\mathrm{TM}}$, they deliver lessons or spontaneously interact with the five virtual students' avatars. The learning objectives are extensive, ranging from classroom management to science or literacy. Iterative virtual rehearsals constitute experiential learning, enhancing pedagogical and interpersonal skills.

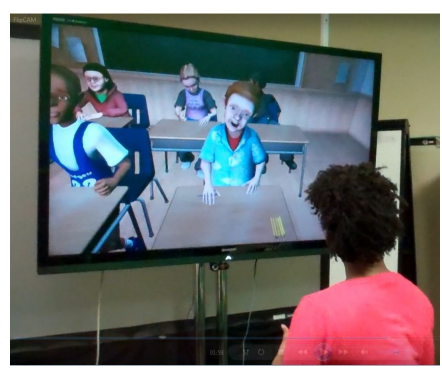

Fig. 1. Teacher delivering a lesson to the TLE TeachLivETM classroom students

\subsection{The Study}

This formative pilot study explores user experience of TLE TeachLivE ${ }^{\mathrm{TM}}$ in regard to the experience of presence, immersion and suspension of disbelief. This research thrust is building toward an investigation of the impact of perceived presence, immersion, and suspension of disbelief on knowledge acquisition and learning transfer. Frequency and fidelity of practice can lead to effective application in the field (Dieker, Hynes, Hughes, \& Smith, 2008; Mahon, Bryant, Brown, \& Kim, 2010). Presence, suspension of disbelief, and immersion were chosen as constructs effective at describing and explaining interpersonal communication and human psychology involved with teaching interpersonal skills (Taylor, 2002; Wagner et. al., 2009). The interrelated nature of these constructs confounds research of them as distinct entities (Harteveld, 2011; Murray, 1997). The reciprocal relationship between presence and immersion was identified as a potential confound to this research, as many scholars erroneously use the terms interchangeably (Witmer \& Singer, 1999; McMahon, 2007; Harteveld, 2011). Embracing and studying the symbiosis between presence, 
suspension of disbelief, and immersion can elucidate significance of these elements on learning.

Because TLE TeachLivE ${ }^{T M}$ is used predominately with educators in K-12 classrooms, the learning objectives were derived from accepted knowledge, skills, and abilities for educators. These behaviors and learning objectives were synthesized from the Bill \& Melinda Gates Foundation's Leveraging High Level Practices and the Measures of Effective Teaching (MET) study (Foundation, 2010).

\section{User Experience}

\subsection{Presence}

The International Society for Presence Research defines presence as, "a psychological state or subjective perception in which even though part or all of an individual's current experience is generated by and/or filtered through human-made technology, part or all of the individual's perception fails to accurately acknowledge the role of the technology in the experience" (2000). Slater and Usoh (1993) distinguish factors of presence as either exogenous or endogenous; exogenous factors are created by the generation of the virtual environment, while endogenous factors are subjective and occur within the user. This distinction calls for analysis of experiences by multiple methods to deepen and enrich understanding of the potential impacts of presence.

\subsection{Suspension of Disbelief}

Wirth \& Saskia synthesize body of knowledge on suspension of disbelief and distill it to the "tolerance of media users towards unreal or implausible content in fictional media" (2005). For the purposes of this study, we expound on this to define suspension of disbelief as the phenomenon in which a participant is able to overlook and even forget the fact that the environment is not natural, but constructed and contrived, in order to enhance engagement, presence, and belief of the experience (Boelstorff, 2011; Dede 2009; Maynes et al. 1996; Jeffries, 2000; Kantor et al. 2000; Hindle 2002; Kushner, 2004; LeRoy et al. 2008; Park, Calvert et al. 2008; Serby, 2011.)

\subsection{Immersion}

Scholars explain immersion as an individual's subjective experience of virtual objects in which they seem to be authentic, which is facilitated by the user's willing suspension of disbelief (Dede, 2009; Witmer \& Singer, 1999). Dede (2009) refines immersion to, "subjective impression that one is participating in a comprehensive, realistic experience" (p.1). The authors of this paper synthesized these to call immersion "an experience in which participants feel not only that they are 'there' with the virtual characters, but also that they have meaningful impact on the environment and entities in the environment" (Dede, 2009; Witmer \& Singer, 1999; McMahan, 2007). 


\section{$3 \quad$ Measuring Learning and Transfer}

Research has been conducted for years attempting to quantify the return on investment in areas of functional fidelity, physical fidelity, and other contributors to user experience (Martin, 1981; Lapkin \& Levvit-Jones, 2011). Some of the methods used to distinguish this relationship include cost-utility analyses that compare the learning outcomes gained from simulators of different levels of fidelity (Lapkin \& Levvit-Jones, 2011; Aldrich 2009). The researchers for this study chose to focus on exploring the relationship between the user's experience and the learning outcomes and transfer of training. In order to test this, we integrated learning objectives into scenarios that could be measured through evaluation before and after the training was delivered. Pedagogical strategies were the education constructs identified for this study: a) specific vs. general praise, b) wait time, and c) higher order questions.

\subsection{Education Constructs to Be Evaluated}

Specific Praise. For the purposes of this study, specific praise refers to positive statements about performance that are explicit in identifying the exact behavior, in order to reinforce and increase the occurrence of the targeted behavior (Kalis, Vannest, \& Parker, 2007; Hawkins \& Heflin, 2010; Feldman, 2003); for example, "Good job showing your work on every question in your assignment". It is a combination of a positive statement linked to the behavior being reinforced such as "Excellent work using a strategy to write your paragraph" (Scheeler, Bruno, Grubb, \& Seavey, 2009).

Wait Time. For the purposes of this study "wait time" is defined as the elapsed time after a teacher ask students a question, and before students respond or the question is rephrased or repeated (Stahl, 1994; Tincani \& Crozier, 2007; Novak, 1963).

Higher Order Questioning. For the purposes of this study "higher-level questioning" is posing questions that allow students to use past experiences, prior knowledge, and previously learned content and relate it to newly learned content in order to create an open ended and well thought out answer (Danielson, 2011; Winne, 1979). Teaching Works, in their report entitled Measures of Effective Teaching, distinguish higher order questioning as strategy of a highly effective teacher (Foundation, 2011).

\section{User Experience Measures}

\subsection{Qualitative User Experience: Interviews}

In the interview the participants elucidated their experience of presence, suspension of disbelief, and immersion through self-report. The interview questions included learning, suspension of disbelief, presence and immersion: 
Overall, how successful do you feel your virtual rehearsal performance was?

How can you tell that the students are engaged or not engaged with you?

How did the virtual students compare to students you encounter in a classroom?

How did the virtual classroom compare to your experience of a physical classroom?

When you were teaching the virtual students, were you able to suspend disbelief?

When teaching the students did you feel like you were in the same physical space as them?

When teaching the students did you feel like you were in the same physical space as them?

How would you describe your use of specific praise?

How would you describe your use of higher order questioning?

How do you feel you used wait time?

\subsection{Qualitative User Experience Measure: Observation}

Observation was utilized by the researchers to interpret the user experience. Presence can be measured by observing reflexive responses to stimuli, such as a participant reaching to catch a ball or flinching or jumping at a stimulus (Sheridan, 1994). This reflexive response can be a physical response, but may also be a reflexive social response to measure presence (Sheridan, 1994). The social responses might include replying to a question, apologizing, or simply saying goodbye before walking away. While this measure could also be refined to an objective measure of the level at which a user has suspended disbelief, this study does not apply it as such a measure.

\subsection{Quantitative User Experience: Questionnaire}

The interview questions for the study were derived from the operational definitions for presence, suspension of disbelief, and immersion. After explaining to participants the meaning of each construct, the researcher then asked them to verbally evaluate the experience according to each of the three. This study utilized interviews and questionnaires that were derived from the Witmer and Singer constructs and their Presence Questionnaire (1998). The Witmer and Singer questionnaire measuring a virtual environment was modified to reflect the needs of a mixed reality environment.

The researchers anticipated relationships between suspension of disbelief and feelings that the students and environment felt real would be revealed by the questionnaire data. The hypotheses that are being tested are:

H1: There will be a relationship between suspension of disbelief and the rating of the environment feeling real.

$\mathrm{H} 2$ : There will be a relationship between suspension of disbelief and the rating of the students feeling real.

\section{Methodology: Mixed Methods Inquiry}

This study began with both subjective measures and objective measures to explore the user experience and the learning outcomes. The participants were practicing and 
pre-service (K-12) teachers in a southeastern state. The time frame of the study was a period of three weeks that began with a baseline observation of their teaching style, followed by three ten minute sessions in TLE TeachLivE ${ }^{\mathrm{TM}}$ that concluded with after action review (AAR) of performance, followed by a final observation in their classroom.

\subsection{Stage 1: Case Study Methodology}

The first stage of this formative research was qualitative, utilizing open-ended user experience interview questions in two case studies investigating the constructs of presence, suspension of disbelief, and immersion. Two middle school teachers were observed in their live classrooms in order to establish a baseline of performance for the constructs (wait time, higher order questioning, and specific praise). The teachers were then immersed in TLE TeachLivE ${ }^{\mathrm{TM}}$ with session objectives of increasing wait time, higher order questioning, and specific praise. Upon completion of each of three 10 minute sessions, the teachers were given feedback in the form of After Action Review (AAR). After the last session in the mixed reality classroom, the teachers were observed again in their live classroom for a post intervention evaluation of performance. The teachers in training were asked open ended questions about their perceptions of the mixed reality classroom environment and the authenticity of the simulated student avatars after their sessions.

In both cases, responses indicated some initial apprehension with the mixed reality classroom environment and the student avatars. They also indicated that the teachers felt that the students in the mixed reality classroom were very much like students that they experience in a live classroom. The participants also indicated that they established emotional relationships with the students such as frustration, empathy, joy, and pride when they succeeded in getting them engaged.

\subsection{TLE TeachLivE ${ }^{\mathrm{TM}}$ Presence Questionnaires}

The first stage of this formative phase of this efficacy research study moved to explore the modified presence questionnaire. The preliminary questionnaire was administered to a convenience sample of 24 pre-service and practicing teachers who were assigned to teach a ten minute lesson in TLE TeachLivETM by the professor for their teaching instruction course. After teaching in TLE TeachLivE they were administered the abbreviated TLE TeachLivE ${ }^{\mathrm{TM}}$ presence questionnaire.

\section{Current Findings}

The preliminary case study supports the idea that virtual learning environments impact learning with targeted practice, authentic scenarios, presence, and suspension of disbelief. 


\subsection{Does the Experience of TeachLivE ${ }^{\mathrm{TM}}$ Effect Learning Outcomes?}

The methods in the baseline research were to observe the teachers as they taught their students in their professional classroom environment. The frequency of the target behaviors, specific praise, higher order questioning, and wait time were measured by two raters, to ensure inter-rater reliability. The teachers were immersed into the environment three separate instances over a period of two weeks, in the TeachLivE ${ }^{\mathrm{TM}}$ classroom as the teacher for 10 minutes. After virtual rehearsal, teachers were given a chance to reflect and also receive feedback in an After Action Review session. Students shared what their perception of their performance was and were met with the reality of how they had actually performed. Each day, the users in the case study improved their performance of the targeted behaviors in the mixed reality classroom environment. The qualitative research revealed user's learning in the lab, as evidenced by the fact that they improved the target behaviors with each iteration of teaching the virtual students. The skills also transferred to the physical classroom with live students, as scores of each of the behaviors were considerably higher in the second measure than the baseline measure of practice in the classroom.

\subsection{Do Students Using TeachLivE ${ }^{\mathrm{TM}}$ Experience Presence?}

The interviews, observations and questionnaires revealed that users are experiencing presence in TLE TeachLivE ${ }^{\mathrm{TM}}$. In post intervention interviews, teachers commented:

\footnotetext{
"I was so nervous."

"I can't believe I made Sean cry; Ifeel so bad,"

"I couldn't get her to put her cell phone away."

"It just feels so real."
}

The observations exposed the characteristics of presence. The participants demonstrated behaviors that indicated presence, such as walking up to the virtual students' approximate locations in the physical space while speaking to them.

Similarly, presence is also evident in the fact that when the session time ends, teachers try to "wrap up the lesson." They say goodbye to the students, and they start planning the next session. This corresponds with the presence measure of reflexive response. These natural responses are transferring to the classroom.

\subsection{Are Students Using TeachLivE ${ }^{\text {TM }}$ Suspending Their Disbelief?}

The case studies and observations of education students indicate that these students are able to suspend their disbelief in the mixed reality classroom. This suspension of disbelief is demonstrated by their emotional response to the virtual students' characteristics and behaviors. The responses in the interview questions asking students if they were suspending their disbelief indicated that they were. This could be observed as teachers rarely treat the simulated students differently than they would real students; which the researcher's code as an indicator of participants having suspended disbelief. 
Statements such as, "Next time I will work at managing Kevin a little better," indicate the suspension of disbelief because the participants are talking about Kevin in a way that ignores the fact that he is a simulated student and not "real".

\subsection{Do Students Using TeachLivE ${ }^{\mathrm{TM}}$ Experience Immersion?}

The interviews revealed that participants felt immersed, according to our definition. Relationships teachers developed with the simulated students and the sense and behavior indicating they felt they could impact the students demonstrated immersion. Likewise, the questionnaire data demonstrated that a majority of users feel the students portray "living kids" (see Table 1). Participants rated the mixed reality classroom as moderately to not at all like a physical classroom. This was consistent with interview responses indicating perceived functional limitations to their practice in the system, such as inability to physically manipulate desks and the limitation of five students.

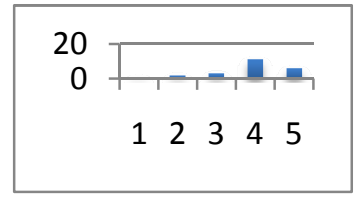

Fig. 2. Rating of the level of realism of the virtual students

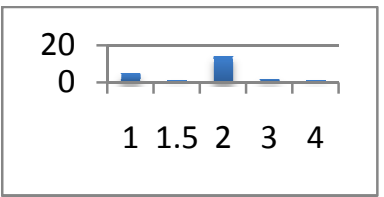

Fig. 3. Rating of the level of realism of the mixed reality classroom

\subsection{Is There an Interaction between Presence, Immersion, and the Suspension of Disbelief?}

This research is focusing primarily on the endogenous factors occurring within the user. Specific constructs investigated in the area of user experience included sense of immersion, presence, and suspension of disbelief. While the questionnaire data did not reveal any significant interaction, the case study interviews support the hypothesis that there is some kind of interaction between these constructs.

\section{Discussion}

These preliminary findings have created a foundation for an additional, larger scale quantitative research study that is currently in process to investigate the relationship between learning and the user experience; specifically suspension of disbelief and 
presence. The long-term research path for this research includes the exploration of the potential impact on presence derived from different interfaces through which the virtual students may manifest themselves.

\subsection{Discussion: Qualitative Findings from Interviews}

The interviews revealed that participants felt a sense of presence in the classroom, but wanted even greater immersion, asking for new features. Teachers indicated they would like to be able to see work samples of each student's actual progress and be able to look over their shoulders. Some of the teachers asked for higher physical fidelity, wanting desks in the room to indicate exactly where the students would be physically located as they navigated the mixed reality classroom. While these experiences were easy to discuss qualitatively, the questionnaire did not differentiate them in the same way.

\subsection{Discussion: Questionnaire Findings}

The questionnaire data did not prove as informative as we hoped, but it has provided a baseline for future qualitative questions. The questionnaire provides only ordinal data in a case where ratio data would be more useful. This could be addressed by changing the scale from a Likert to semantic differential, in order to give the participants shared reference criterion. For instance, would a participant rating of highest realism for the avatar represent a sense of its being indistinguishable from "living" children or the most realistic that the participant had witnessed to date?

Finally, the sample of 24 participants who completed the questionnaire was not large enough to adequately represent the population. This was further confounded by the fact that half of the participants had teaching experience in a physical classroom while the other half had no experience. This was confounding as it effectively rendered our sampling as being two samples of 12 participants in each group.

\subsection{Future Research}

Future research in this area is currently being conducted. The next phase of the research into the efficacy of the TeachLivE ${ }^{\mathrm{TM}}$ simulator is a large-scale study that will include 200 practicing teachers across the United States. The control group of this study will have their performance evaluated at the baseline and again after three weeks. TLE TeachLivE ${ }^{\mathrm{TM}}$ users will complete the revised presence questionnaire.

Acknowledgements. We would like to acknowledge the Bill \& Melinda Gates Foundation, whose gift in part supported the TeachLivE ${ }^{\mathrm{TM}}$ team and the execution of this study, and the National Science Foundation (CNS1051067) whose support contributed to the technical infrastructure. We would like to acknowledge the other principle investigators (PIs) of TLE TeachLivETM, Drs. Lisa Dieker, and Michael Hynes. We would also like to acknowledge the work of Dr. Carrie Straub, the coordinator of the second phase of TeachLivE ${ }^{\mathrm{TM}}$ research. Finally, we would like to acknowledge 
the efforts of our partner universities, whose reflections facilitated development and yielded consensus to the areas of highest concern. Opinions, findings, and conclusions or expressed in this material do not necessarily reflect the views of these supporters.

\section{References}

1. Aldrich, C.: Learning Online with Games, Simulations, and Virtual Worlds: Strategies for Online Instruction. Jossey-Bass, San Francisco (2009)

2. Bailenson, J., Beall, A., Loomis, J., Blascovich, J., Turk, M.: Transformed Social Interaction: Decoupling Representation from Behavior and Form in Collaborative Virtual environments. Presence: Teleoperators\& Virtual Environments 13(4), 428-441 (2004)

3. Boellstorff, T.: Virtuality. A Companion to the Anthropology of the Body and Embodiment, 504-520 (2011)

4. Dieker, L., Hynes, M., Hughes, C., Smith, E.: Implications of Mixed Reality and Simulation Technologies on Special Education and Teacher Preparation. Focus on Exceptional Children 40(6), 5 (2008)

5. Foundation, B.M.G.: Learning about Teaching: Initial Findings from the Measures of Effective Teaching Project. Bill \& Melinda Gates Foundation, MET Project (2010)

6. Harteveld, C.: Triadic Game Design Balancing Reality, Meaning and Play (2011)

7. International Society for Presence Research.: The Concept of Presence: Explication Statement (2000), http: / / ispr . info/ (retrieved January 2012)

8. Kalis, T., Vannest, K., Parker, R.: Praise Counts: Using Self-Monitoring to Increase Effective Teaching Practices. Preventing School Failure 5193, 20-27 (2007)

9. Mahon, J., Bryant, B., Brown, B., Kim, M.: Using Second Life to Enhance Classroom Management Practice in Teacher Education. Educational Media International 47(2) (2010)

10. McMahan, A.: Immersion, Engagement, and Presence: A Method for Analyzing 3D Videogames. In: The Video Game Theory Reader, pp. 67-86. Routledge (2003)

11. Murray, J.: Hamlet on the Holodeck: The Future of Narrative in Cyberspace, pp. 98-99. The MIT Press, Cambridge (1997)

12. Sheridan, T.: Further Musings on the Psychophysics of Presence. Paper presented at the Systems, Man, and Cybernetics. IEEE Conference on Humans, Inf. and Technology (1994)

13. Slater, M., Usoh, M.: Presence in Immersive Virtual Environments. In: IEEE Virtual Reality Annual International Symposium, Seattle, Washington, pp. 90-96 (1993)

14. Slater, M.: Measuring Presence: A Response to the Witmer and Singer Presence Questionnaire. Presence: Teleoperators\& Virtual Environments 8(5), 560-565 (1999)

15. Witmer, B., Singer, M.: Measuring Presence in Virtual Environments: A Presence Questionnaire. Presence: Teleoperators\& Virtual Environments 7(3), 225-240 (1998)

16. Wirth, W., Boecking, S.: Towards conceptualizing suspension of disbelief for communication research: International Communication Association (2005)

17. Nakano, Y.I., Ishii, R.: Estimating User's Engagement from Eye-Gaze Behaviors in Human-Agent Conversations. In: Proceedings of the 15th International Conference on Intelligent user Interfaces (IUI 2010), pp. 139-148. ACM, New York (2010) 